\title{
Comparative Analysis of a High Pass Finite Impulse Response (FIR) Filter Designed and Simulated by Window Methods
}

\section{${ }^{* 1}$ SHEHU, NM; ${ }^{2}$ BARAYA, JT; ${ }^{3}$ SANI, MH}

\author{
${ }^{*}$ Department of Physics, Bayero University, Kano, Nigeria \\ ${ }^{2}$ Department of Physics, Federal University, Dutsin-Ma, Katsina State, Nigeria \\ ${ }^{3}$ Department of Science Laboratory Technology, Kano State Polytechnic Nigeria \\ *Corresponding Author Email: nmshehu.phy@buk.edu.ng, Tel.: +234-8038860203
}

\begin{abstract}
The design of digital filters is a deceptively complex topic. Although filters are easily understood and calculated, the practical challenges of their design and implementation are significant and are the subject of much advanced research. In this research paper, a high pass FIR filter is designed and simulated using Gaussian, Taylor and Hanning windows. The three windows are compared on the basis of their magnitude responses, step responses, impulse responses and phase responses and the results are presented. The comparative result shows that the proposed design of Taylor window is the most efficient of the three windows.
\end{abstract}

DOI: $\underline{\text { https://dx.doi.org/10.4314/jasem.v23i4.23 }}$

Copyright: Copyright $\odot 2019$ Shehu et al. This is an open access article distributed under the Creative Commons Attribution License (CCL), which permits unrestricted use, distribution, and reproduction in any medium, provided the original work is properly cited.

Dates: Received: 17 February 2019; Revised: 29 March 2019; Accepted 22 April 2019

Keywords: High Pass Filter, Window Method, Gaussian Method, Impulse response

In signal processing, a filter is a device or process that removes some unwanted components or features from a signal. Filtering is a class of signal processing, the defining feature of filters being the complete or partial suppression of some aspect of the signal. Most often, this means removing some frequencies or frequency bands. However, filters do not exclusively act in the frequency domain; especially in the field of image processing many other targets for filtering exist. Correlations can be removed for certain frequency components and not for others without having to act in the frequency domain. Filters are widely used in electronics and telecommunication, in radio, television, audio recording, radar, control systems, music synthesis, image processing, and computer graphics. A digital filter system usually consists of an analog-to-digital converter to sample the input signal, followed by a microprocessor and some peripheral components such as memory to store data and filter coefficients etc., finally a digital-to-analog converter to complete the output stage. Program Instructions (software) running on the microprocessor implement the digital filter by performing the necessary mathematical operations on the numbers received from the ADC. In some high performance applications, an FPGA or ASIC is used instead of a general purpose microprocessor, or a specialized DSP with specific paralleled architecture for expediting operations such as filtering. (Shehu et al., 2016)
FIR Filter: Finite impulse response (FIR) filter is a filter whose impulse response (or response to any finite length input) is of finite duration, because it settles to zero in finite time. A finite impulse response (FIR) filter is a filter structure that can be used to implement almost any sort of frequency response digitally. An FIR filter is usually implemented by using a series of delays, multipliers and adders to create the filter's output. FIR filters also known as feed forward or non-recursive, or transversal filters. The unit impulse response is finite; so FIR filters are stable system (Mahto et.al, 2017).

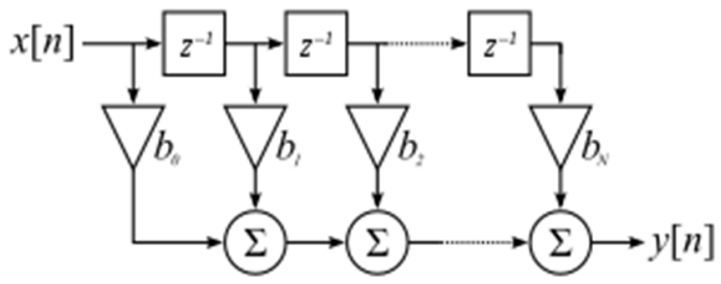

Fig.1: Block diagram of FIR Filter

FIR Filter Design: It is possible to design a Finite Impulse Response (FIR) filter from an Infinite Impulse Response (IIR) by many methods. One of the most commonly used methods is the window method which provides a room for truncating an infinite impulse

*Corresponding Author Email: nmshehu.phy@buk.edu.ng 
response. There are various types of window methods which could be used to design an FIR filter. These include: Taylor, Kaiser, Hamming, Hanning, Gaussian and so on.

Window Function: In signal processing and statistics, a window function (also known as an apodization function or tapering function ) (Weinstein, 2003) is a mathematical function that is zero-valued outside of some chosen interval, normally symmetric around the middle of the interval, usually near a maximum in the middle, and usually tapering away from the middle. Mathematically, when another function or waveform/data-sequence is "multiplied" by a window function, the product is also zero-valued outside the interval: all that is left is the part where they overlap, the "view through the window". Equivalently, and in actual practice, the segment of data within the window is first isolated, and then only that data is multiplied by the window function values. Thus, tapering, not segmentation, is the main purpose of window functions. The windowing method requires minimum amount of computational effort, so window method is simple to implement. For the given window, the maximum amplitude of ripple in the filter response is fixed. Thus the stop band attenuation is fixed in the given window, but there is some drawback also of this method. The design of FIR filter is not flexible (Weinstein, 2003). In this paper, Gaussian, Taylor and Hanning methods are used to design filter.

Gaussian Window: The Fourier transform of a Gaussian is also a Gaussian (it is an Eigen of the Fourier transform). Since the Gaussian function extends to infinity, it must either be truncated at the ends of the window, or itself windowed with another zero-ended window. Since the $\log$ of a Gaussian produces a parabola, this can be used for nearly exact quadratic interpolation in frequency estimation.

$$
\begin{aligned}
& w(n)=e^{-\frac{1}{2}\left(\frac{n-(N-1) / 2}{\sigma(N-1) / 2}\right)^{2}} \\
& \sigma \leq 0.5
\end{aligned}
$$

The standard deviation of the Gaussian function is $\sigma$ $(N-1) / 2$ sampling periods.

Taylor Window: Taylor windows are similar to Chebyshev windows. While a Chebyshev window has the narrowest possible main lobe for a specified side lobe level, a Taylor window allows you to make tradeoffs between the main lobe width and side lobe level. The Taylor distribution avoids edge discontinuities, so Taylor window side lobes decrease monotonically. Taylor window coefficients are not normalized. Taylor windows are typically used in radar applications, such as weighting synthetic aperture radar images and antenna design. $\mathrm{w}=$ taylorwin (n) returns an n-point Taylor window in a column vector $w$. The values in this vector are the window weights or coefficients. $n$ must be a positive integer. The default value for the number of approximately equal height sidelobes (n,bar) is 4 and for the maximum sidelobe level ( $\mathrm{sll})$ is -30 . $\mathrm{W}=$ taylorswin (n,bar) returns an n-point Taylor window with nbar nearly constant-level sidelobes adjacent to the mainlobe. These sidelobes are "nearly constantlevel" because some decay occurs in the transition region. nbar must be a positive integer (Carrara et al., 1995).

Hanning Window: The hanning window is a raised cosine window. The hanning window is defined as (Khatun, 2018)

$$
W(n)=\left\{\begin{aligned}
0.5-0.5 & \cos \frac{z \pi n}{M-1}, \text { for } n \\
0, \quad \text { elsewere } & 0 \text { to } M-1 \quad \ldots 2
\end{aligned}\right.
$$

\section{RESULTS AND DISCUSSION}

In designing the high pass FIR filter using the Hamming, Gaussian and Taylor window methods, the

\begin{tabular}{|c|c|c|c|c|c|c|}
\hline Window & $\begin{array}{l}\text { Sampling } \\
\text { frequency } \\
\text { (fs)(MHz) }\end{array}$ & $\begin{array}{l}\text { Cut off } \\
\text { freq uency } \\
\text { (fc) }(\mathrm{MHz})\end{array}$ & $\begin{array}{l}\mathrm{Ord} \\
(\mathrm{N})\end{array}$ & r Nbar & $\begin{array}{l}\text { Sidelobe } \\
\text { level }\end{array}$ & $\begin{array}{l}\text { Alpha } \\
\text { (a) }\end{array}$ \\
\hline Gaussian & 20 & 3 & 50 & - & - & 2.5 \\
\hline $\begin{array}{l}\text { Tayior } \\
\text { Hanning }\end{array}$ & $\begin{array}{l}20 \\
20\end{array}$ & $\begin{array}{l}3 \\
3\end{array}$ & $\begin{array}{l}50 \\
50\end{array}$ & $\begin{array}{l}4 \\
-\end{array}$ & 30 & - \\
\hline
\end{tabular}
parameters used are shown in Stable1 below:

Table 1: Parameter Specifications

Using the specifications of table1 above, Gaussian, Taylor and Hanning Windows are simulated by MATLAB R2013b and the results are presented in the figures 1 to 16 .

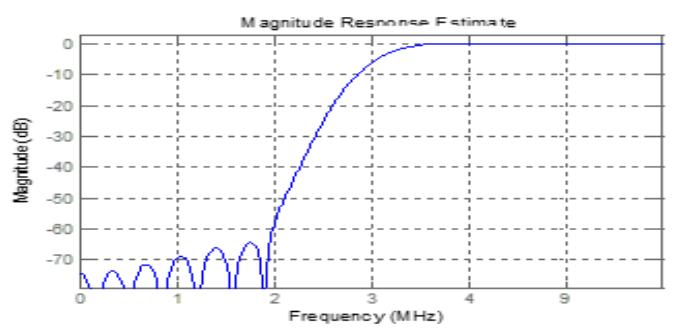

Fig. 2: Magnitude response of Gaussian window

Gaussian Window Simulation: From the fig. 2 above, the magnitude response of Gaussian window cuts at $1.91 \mathrm{MHz}$ which is far away from the cut off frequency ( $3 \mathrm{MHz}$ ). The gap between this magnitude response and the cut off frequency is $1.09 \mathrm{MHz}$. 


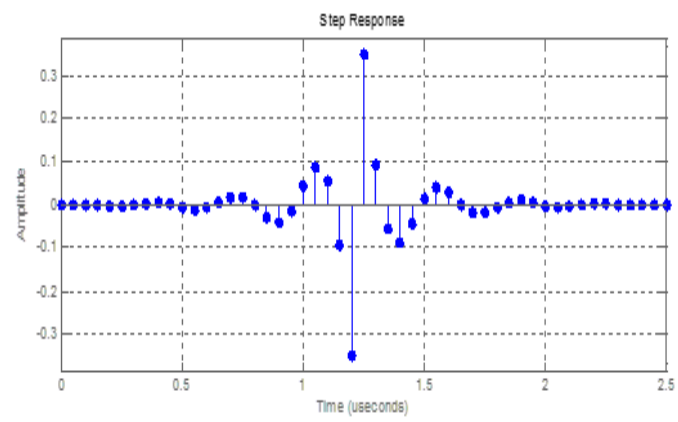

Fig. 3: Step response of Gaussian window

The fig. 3 above shows an over-response of the step response for the Gaussian window

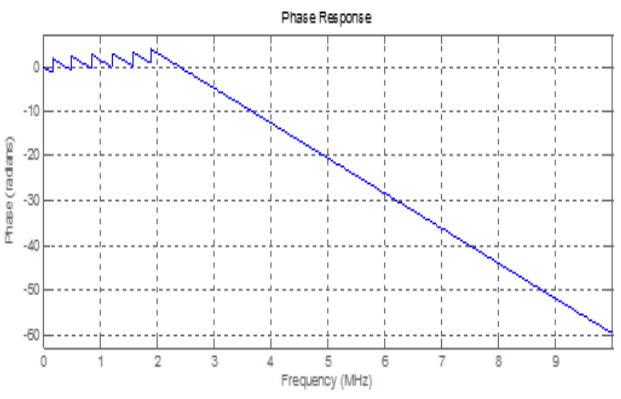

Fig. 4: Phase response of Gaussian window

Observing fig. 4 above, the phase of the Gaussian window changes at $1.93 \mathrm{MHz}$.

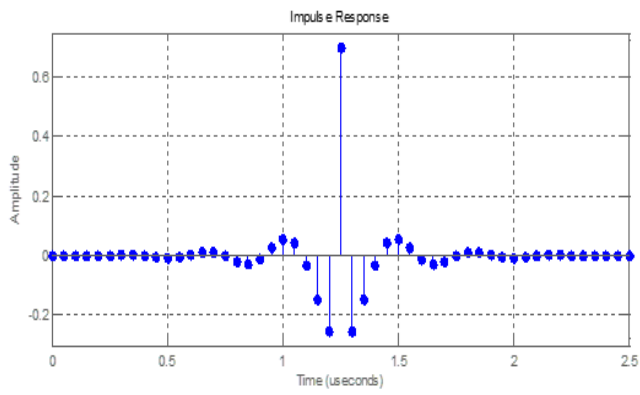

Fig. 5: Impulse response of Gaussian window

Fig. 5 above shows that the impulse of the Gaussian window has over-responded.

Taylor Window Simulation: From the fig. 6 above, the magnitude response of the Taylor window cuts at 2.46 $\mathrm{MHz}$ which is a bit away from the cut off frequency (3 $\mathrm{MHz}$ ) but closer compared with the magnitude response of Gaussian window. The gap between this magnitude response and the cut off frequency is 0.54 Mhz. From the fig. 7 above, we can see that there is an over-response of the step response for Taylor window. From fig. 8 above, we can see an over-response of the impulse response for Taylor window. From fig. 9 above, the phase change of the Taylor window occurs at $2.45 \mathrm{MHz}$.

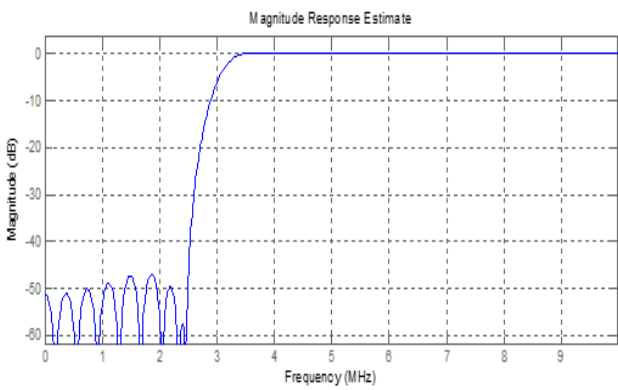

Fig. 6: Magnitude response of Taylor window

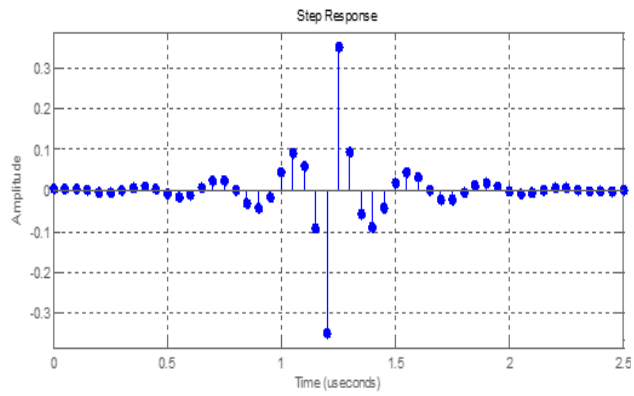

Fig. 7: Step response of Taylor window

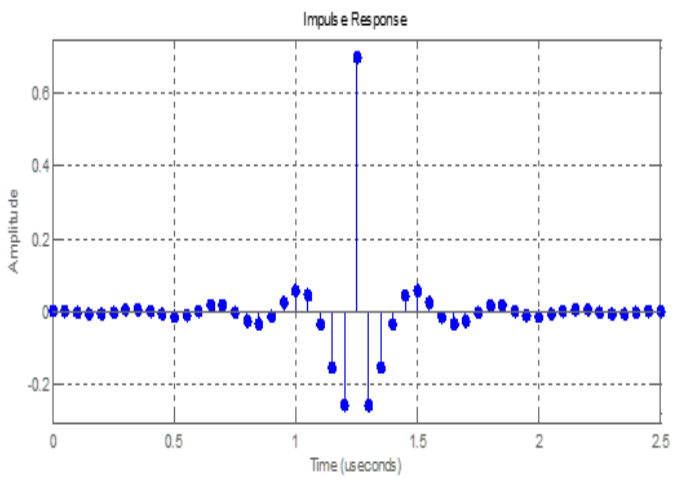

Fig. 8: Impulse response of Taylor window

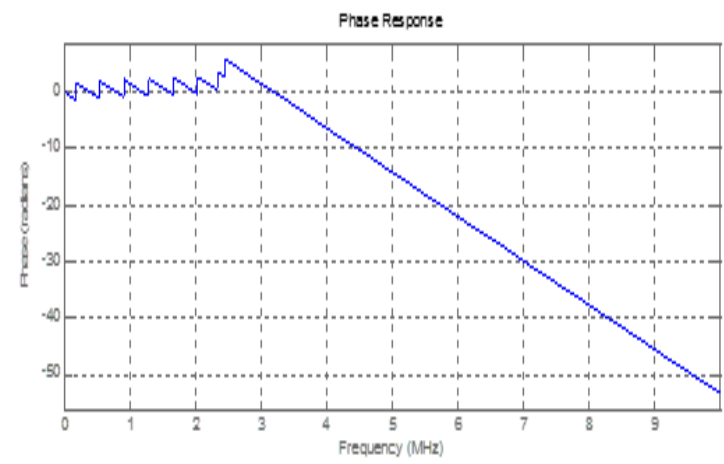

Fig. 9: Phase response of Taylor window

Hanning Window Simulation: From the fig. 10 above, the magnitude response of Hanning window cuts at 2.34 MHz which is a bit away from the cut off 
frequency (3 MHz) but closer compared with the magnitude response of Gaussian window. The gap between this magnitude response and the cut off frequency is $0.66 \mathrm{MHz}$.

Magnitude Resporse Estimate

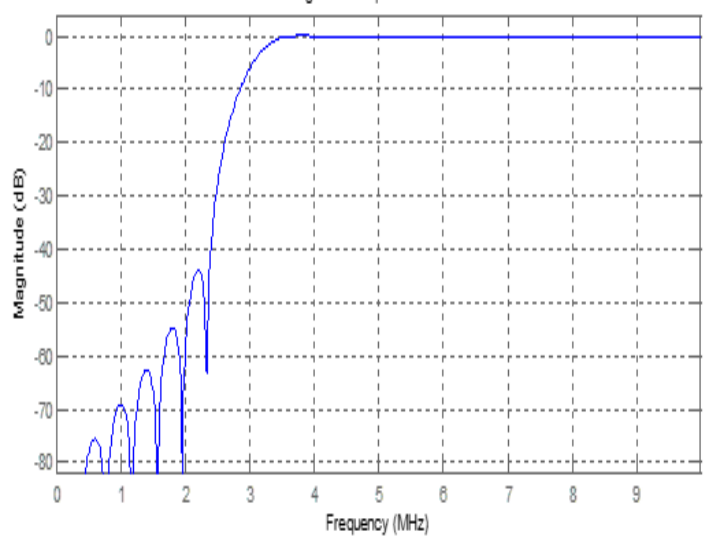

Fig. 10: Magnitude response of Hanning window

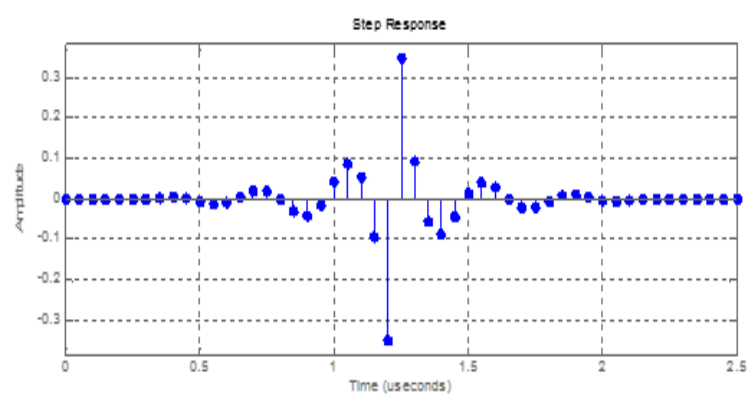

Fig. 11: Step response of Hanning window

From fig. 11 above, we can see that there is an overresponse.

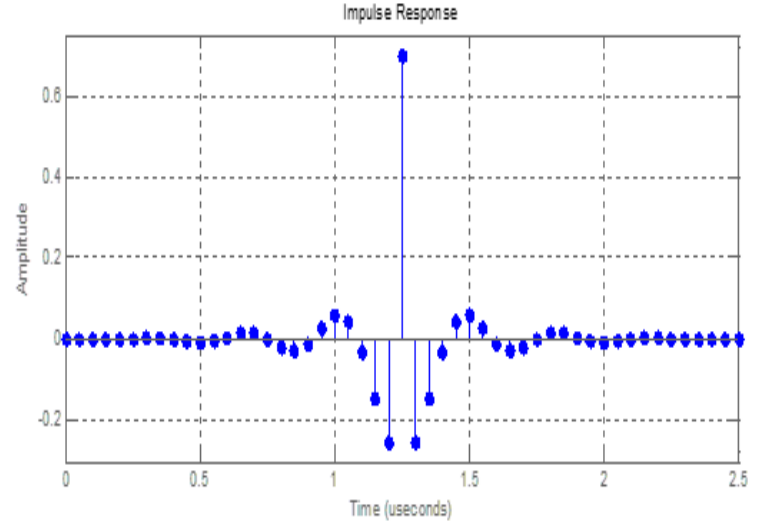

Fig. 12: Impulse response of Hanning window

From fig. 12 above, we can see that there is an overresponse. Observing fig. 13 above the phase of the Gaussian window changes at $2.34 \mathrm{MHz}$.

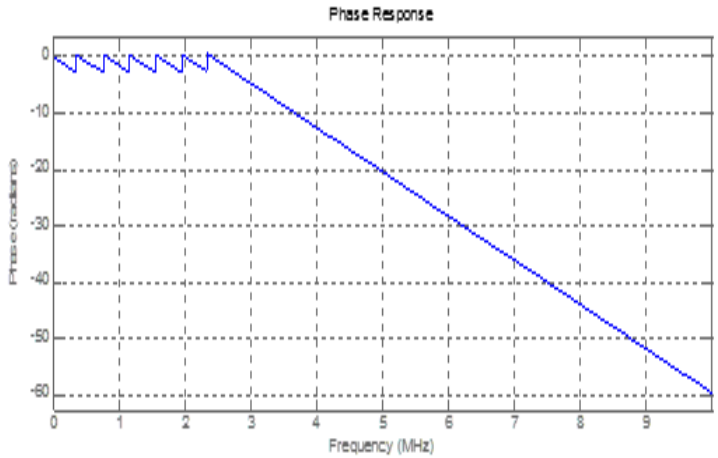

Fig.13: Phase response of Hanning window

Comparative Analysis: Various responses of Gaussian, Taylor and Hanning Windows are plotted and shown on a single figure for comparison as in fig. 14-17.

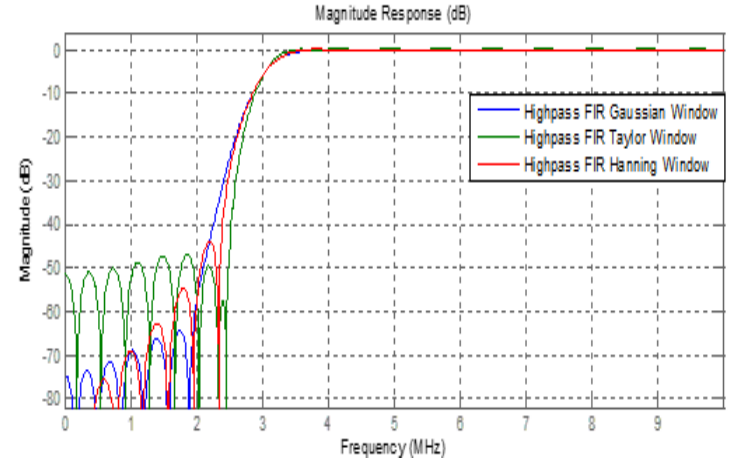

Fig. 14: Comparison among Magnitude responses

In fig. 14 above, the magnitude responses of the Gaussian, Taylor and Hanning windows are plotted together on a single figure for comparison. From the figure, it can be closely viewed that Gaussian window cuts at $1.91 \mathrm{MHz}$, Taylor window cuts at $2.46 \mathrm{MHz}$ and Hanning cuts at $2.34 \mathrm{MHz}$. On the basis of the magnitude response comparison, Taylor which cuts at $2.46 \mathrm{MHz}$ is the closest to the specified cut off frequency of the remaining two windows.

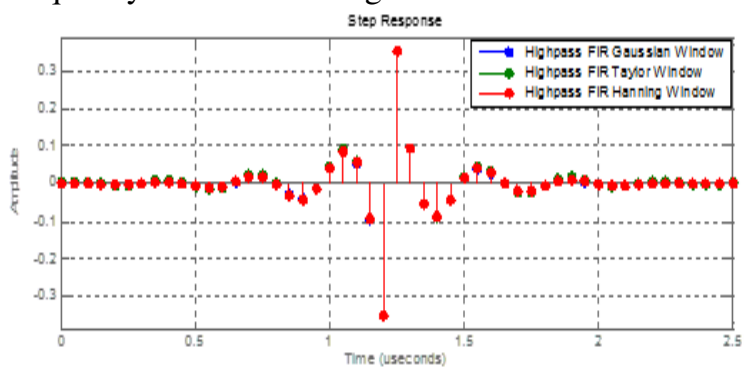

Fig. 15: Comparison among Step responses

In fig. 15 above, the step responses of the Gaussian, Taylor and Hanning windows are plotted together for comparison. From the figure, it can be observed that there is an over-response of the three responses. In fig. 
16 above, the impulse responses of the Gaussian, Taylor and Hanning windows are plotted together for comparison. From the figure, it can be observed that the there is an over-response of the three responses.

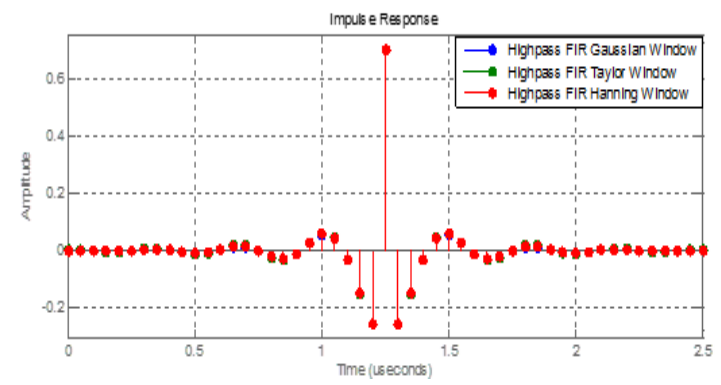

Fig. 16: Comparison among Impulse responses

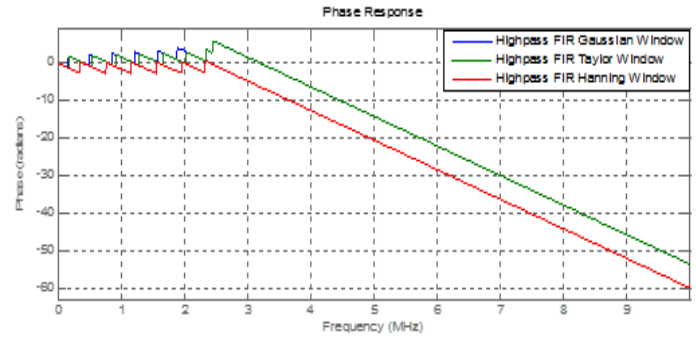

Fig. 17: Comparison among Phase responses

In fig. 17 above, the phase responses of the Gaussian, Taylor and Hanning windows are plotted together for comparison. From the figure, it can be observed that the there is an over-response of the three responses.

\begin{tabular}{lllll}
\multicolumn{5}{c}{ Table 2: Comparing among Gaussian, Taylor and Hanning Windows } \\
\hline S/N & Parameter & Gaussian & Taylor & Hanning \\
\hline 1 & Magnitude Response & Cuts off at 1.91MHz & Cuts off at 2.46MHz & Cuts off at 2.32MHz \\
2 & Phase Response & Phase change at $1.93 \mathrm{MHz}$ & Phase change at 2.45MHz & Phase change at 2.34MHz \\
3 & Impulse Response & Over response & Over response & Over response \\
4 & Step Response & Over response & Over response & Over response \\
5 & Filter Coefficient & 22 numerators & 20 numerators & 23 numerators \\
6 & Leakage Factor & $0.01 \%$ & $0.44 \%$ & $0.05 \%$ \\
7 & Relative Sidelobe Attenuation & $-44.3 \mathrm{~dB}$ & $-30.3 \mathrm{~dB}$ & $-31.5 \mathrm{~dB}$ \\
8 & Mainlobe width, (-3dB) & 0.054688 & 0.042969 & 0.058594 \\
9 & Stable & Yes & Yes & Yes \\
\hline
\end{tabular}

From the values in the table it is shown that Taylor cuts at $2.46 \mathrm{MHz}$ which is nearer to the specified cut off frequency $(3 \mathrm{MHz})$ chosen in the design. The impulse and step responses are the same. The phase response of Taylor and Hanning are nearly the same which change at $2.45 \mathrm{MHz}$ and $2.34 \mathrm{MHz}$ respectively with Gaussian which has phase change at $1.93 \mathrm{MHz}$.

Conclusion: A high pass FIR filter has been designed and simulated using Gaussian, Taylor and Hanning window methods in this research paper. In table 2 magnitude response, impulse response, step response and phase response have been compared at the same sampling frequency, cut off frequency and order. Based on the results obtained, it is concluded that the proposed design of Taylor window is the most efficient of the three windows.

\section{REFERENCES}

Shehu, NM; Gidado, AS; Wudil, YS; Gora, UA (2016). Performance Analysis of FIR Low Pass Filter Design using Blackman and Flat Top Window Methods. Inter. J. Engineer. Sci. Comp. 6(4), 3659-3665.

Mahto, A; Chaubey, M; Rahi, PK (2017). Optimal Design of Low Pass Filter by Hamming, Hanning and Kaiser Technique. Inter. J. Adv. Res. Comp. Comm. Engineer. 6(6), 443-452.
Weinstein, E (2003). CRC Concise Encyclopedia of Mathematics. CRC Press. ISBN 1-58488-347-2.

Carrara, WG; Majewski, RM; Goodman, RS (1995). Spotlight Synthetic Aperture Radar: Signal Processing Algorithms, Artech House Publishers, Boston, Appendix D.2.

Khatun, M (2018). Design Technique of High-pass Filter using different window. Inter. J. Engineer. Comp. Sci. 7(2), 23585-23589. 